Article

\title{
Different Fluorophore Labeling Strategies and Designs Affect Millisecond Kinetics of DNA Hairpins
}

\author{
Andreas Hartmann ${ }^{1, \dagger}$, Georg Krainer ${ }^{1,2, \dagger}$ and Michael Schlierf ${ }^{1, *}$
}

1 B CUBE, Center for Molecular Bioengineering, Technische Universität Dresden, Dresden 01307 , Germany; E-Mails: hartmann@bcube-dresden.de (A.H.); krainer@bcube-dresden.de (G.K.);

Molecular Biophysics, University of Kaiserslautern, Kaiserslautern 67663, Germany

$\dagger$ These authors contributed equally to this work.

* Author to whom correspondence should be addressed; E-Mail: schlierf@bcube-dresden.de; Tel.: +49-351-4634-3050.

Received: 18 July 2014; in revised form: 21 August 2014 / Accepted: 26 August 2014 /

Published: 3 September 2014

\begin{abstract}
Changes in molecular conformations are one of the major driving forces of complex biological processes. Many studies based on single-molecule techniques have shed light on conformational dynamics and contributed to a better understanding of living matter. In particular, single-molecule FRET experiments have revealed unprecedented information at various time scales varying from milliseconds to seconds. The choice and the attachment of fluorophores is a pivotal requirement for single-molecule FRET experiments. One particularly well-studied millisecond conformational change is the opening and closing of DNA hairpin structures. In this study, we addressed the influence of base- and terminal-labeled fluorophores as well as the fluorophore DNA interactions on the extracted kinetic information of the DNA hairpin. Gibbs free energies varied from $\Delta G^{0}=-3.6 \mathrm{~kJ} / \mathrm{mol}$ to $\Delta G^{0}=-0.2 \mathrm{~kJ} / \mathrm{mol}$ for the identical DNA hairpin modifying only the labeling scheme and design of the DNA sample. In general, the base-labeled DNA hairpin is significantly destabilized compared to the terminal-labeled DNA hairpin and fluorophore DNA interactions additionally stabilize the closed state of the DNA hairpin. Careful controls and variations of fluorophore attachment chemistry are essential for a mostly undisturbed measurement of the underlying energy landscape of biomolecules.
\end{abstract}

Keywords: DNA hairpin; labeling strategy; single-molecule FRET; hairpin design 


\section{Introduction}

The analysis of biomolecular conformational dynamics is of fundamental importance for a better understanding of complex biological processes. Single-molecule techniques have the power to directly resolve distributions and heterogeneities of individual biomolecular complexes underlying the ensemble average and follow unsynchronized dynamics that might remain masked when large numbers of molecules are probed simultaneously [1,2]. In particular, single-molecule Förster Resonance Energy Transfer (smFRET) has become a powerful and popular tool to explore and quantify conformational dynamics of nucleic acids [3-22] and proteins [23-35]. smFRET experiments quantify the energy transfer efficiency between a single donor and acceptor fluorophore pair. The non-radiative energy transfer strongly depends on the inter-fluorophore distance and has a characteristic length (Förster radius) on the order of a few nanometers, thus, matching the length scales of most biomolecules. smFRET can be used as a molecular ruler for biological systems and is a complementary technique to methodologies, such as X-ray crystallography, NMR or electron microscopy, supplementing structural information with dynamic content. Nowadays, two conceptually different approaches are frequently used for smFRET experiments [36]: surface immobilized studies and confocal spectroscopy with freely-diffusing molecules. Surface immobilized FRET studies based on total-internal-reflection fluorescence (TIRF) microscopy allow the observation of slow conformational dynamics (tens of milliseconds to minutes) of individual molecules, while confocal spectroscopy combined with time-correlated single-photon counting (TCSPC) on both, freely-diffusing and surface immobilized molecules, enables the observation of distinct populations with fast interconversion dynamics (microseconds to milliseconds) [37].

Among the studies on dynamic biomolecules in living systems at the single-molecule level, investigations of the conformational changes of DNA and RNA hairpins have become one of the main topics. Hairpins display a relatively simple structural element that consists of inverse repeats of single-stranded DNA connected by a non-complementary loop region. Depending on their stem length, hairpins constantly undergo changes between folded (closed) and unfolded (open) conformations with different kinetic rates. Nucleic-acid hairpins are highly conserved genetic elements involved in DNA recombination, gene transcription and DNA replication [38]. RNA hairpins are, for example, involved in regulating protein translation by forming temperature-responsive hairpin structures $[39,40]$. Moreover, hairpins are also used in nanotechnological applications as biosensors and reporters (e.g., as molecular beacons or anti-sense dugs) in diagnostics and drug development [41,42]. Thus, a fundamental understanding of hairpin structures and their underlying energy landscape is essential. Opening and closing kinetics of hairpins have been studied in detail previously by means of smFRET and complementary fluorescence techniques reported to depend strongly on sequence, stem length, salt concentration and temperature [9-17,43-51]. In these studies two fundamentally different hairpin designs were used. The first design is a minimal model consisting of a single-stranded DNA with a central loop and complementary sequences at the ends, resembling molecular beacons. Fluorophores were attached in this design to both, the 3'- and 5'-end (e.g., [9,10]). The second design (e.g., [11-13,15,16] uses an additional single-stranded DNA to separate the donor and acceptor fluorophore to avoid dye-dye stacking that could lead to quenching and additional blinking [52]. This 
design creates a proximal stem that contains the complementary hairpin sequence and a long distal stem that contains the donor or acceptor fluorophore (Figure 1A).

Figure 1. (A) Schematic for the conformational fluctuation of the stem-loop DNA hairpin between closed and open states with the characteristic opening rate and closing rate, $k_{\text {open, }}$, $k_{\text {close }}$, respectively .The basic DNA hairpin consists of a T21 loop and a 5 bp proximal stem (5'-AACCA-3'), followed by a 25-30 bp long distal stem. All constructs were labeled at position 26 with ATTO532 and at position 36 with ATTO647N; (B-G) FRET efficiency histograms of the six different DNA hairpins after removal of donor-only and acceptor-only populations and photobleaching events (see text).

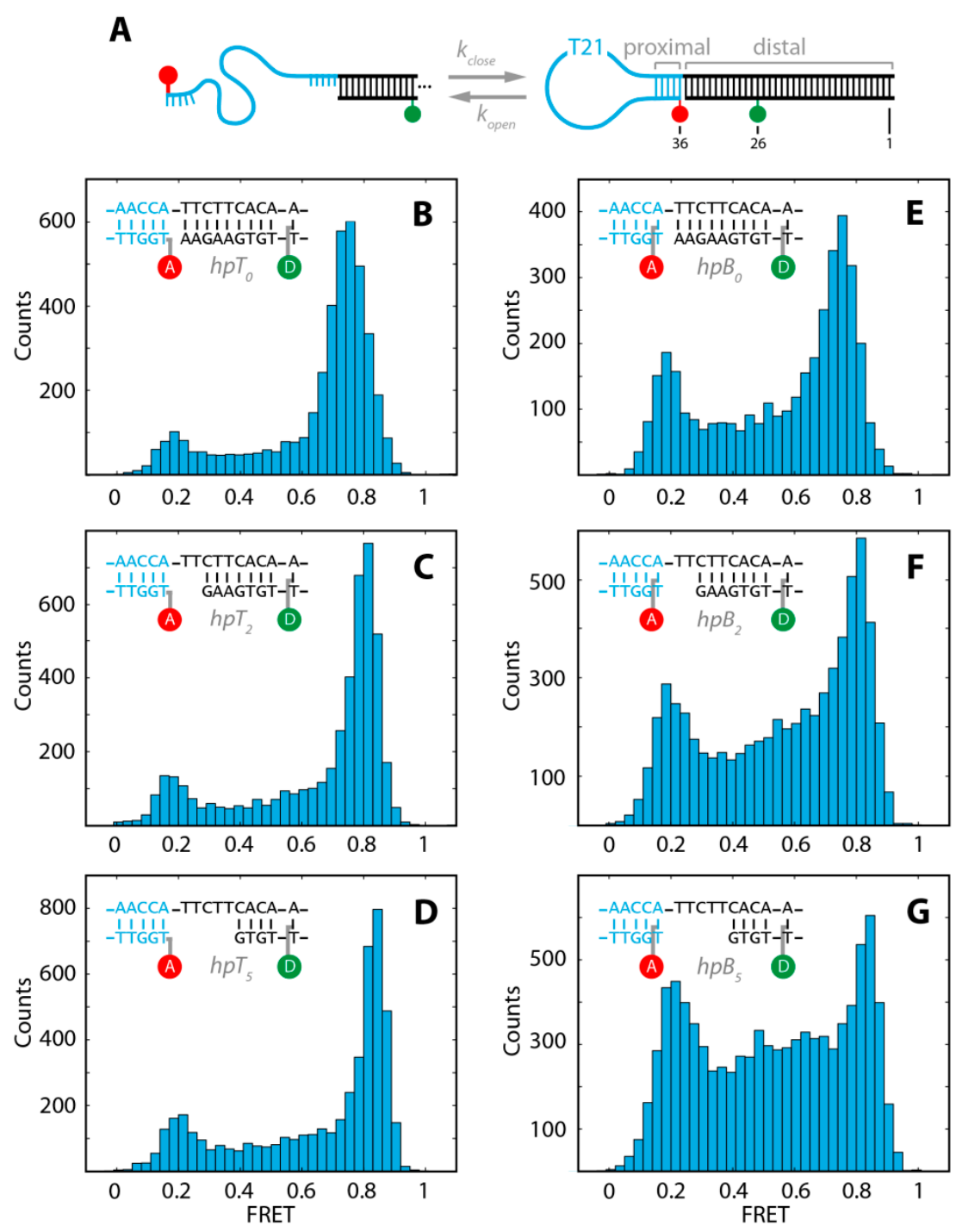

The attachment of fluorescent probes, preferentially organic fluorophores, is a pivotal step to report on conformational changes between the open and closed state in hairpins by monitoring differences in the FRET efficiency. Among the wealth of different labeling approaches available for site-specific modification of DNA and RNA nucleotides the most commonly used strategies comprise either conjugation of the fluorophores to the 5 '- or 3 '-end of the nucleotide (terminal labeling) or at internal positions of nucleobase derivatives (base labeling). For terminal labeling on the 5'- or 3'-ends, fluorophores are covalently linked to the terminal phosphate group. This conjugation is accomplished 
mostly either via phosphoramitide-based chemistry or via an amine reaction to an amino-functionalized aliphatic linker attached to the terminal phosphate group. In base-labeling, nucleotide bases of pyrimidines (e.g., thymidines) modified with an aliphatic amino linker attached to the C5 ring are conjugated with amine-reactive functionalized fluorophores.

In respect to the different labeling strategies, extreme care has to be taken not to perturb residues or regions that might be involved in the formation of interactions and thereby severely destabilize the folded structure. This in turn may interfere with the actual conformational dynamics of the biomolecule to be observed and, thus, change its characteristics of the underlying energy landscape $[14,53]$. Moreover, fluorophore properties can be altered depended on the local microenvironment of the biomolecule [54-59].

To date, little is known about the influence of distinct labeling strategies and the distal stem on the dynamic equilibrium between the folded and unfolded conformations. Changes of the physicochemical properties of DNA or RNA introduced by different positioning of the labels and distal stems, could potentially affect the thermodynamic stability of hybridization and, consequently, the underlying energy landscape. A solid interpretation of these changes, however, requires detailed knowledge of the influence of the fluorophores on the conformational transitions.

In this study, we addressed the influence of the labeling strategies and the design of the distal stem on the millisecond kinetics of a DNA hairpin using diffusion-based pulsed interleaved excitation smFRET burst analysis with multiparameter fluorescence detection [60-63]. Millisecond kinetics of DNA hairpin formation have been extracted using recently developed analysis methods based on the approximation of the FRET efficiency distribution by a sum of three-Gaussian functions $[64,65]$. We found that base-labeled hairpins are destabilized compared to the terminal-labeled hairpins and, thus, can lead to a misinterpretation of the state and rate constants. Additionally, distal stem designs stabilized the closed hairpin conformation significantly.

\section{Results and Discussion}

\subsection{DNA Hairpins with Millisecond Kinetics}

In order to investigate the influence of different labeling strategies and distal stem designs for a DNA hairpin with millisecond kinetics, we used a hairpin construct (Figure 1A) with a T21 loop region and a 5 bp proximal stem, containing a GC content of $40 \%$. The distal stem length was varied between $25 \mathrm{bp}$ and $30 \mathrm{bp}$ to introduce a gap of zero, two or five nucleotides between the proximal and distal stem (insets in Figure 1B-G). The 5 bp sequence of the proximal stem and the length of the loop were chosen such that the opening and closing kinetics are on the millisecond time-regime [12,13,15-17]. Conformational changes were probed with the FRET pair ATTO532 and ATTO647N as donor and acceptor fluorophores, respectively. The donor fluorophore was attached to the distal stem via base labeling (position 26). The acceptor was conjugated to the proximal stem at the 5'-end (position 36) either via base (hpB) or terminal labeling (hpT) (Figure 1A). In the closed (folded) conformation the distance of donor and acceptor result in a high efficiency of energy transfer (high FRET), in the open (unfolded) conformation the increased distance between donor and acceptor lead to a low efficiency of energy transfer (low FRET). The two differently labeled proximal stems in combination with the three different distal stem designs result in six constructs $\left(\mathrm{hpB}_{0}, \mathrm{hpB}_{2}, \mathrm{hpB}_{5}, \mathrm{hpT}_{0}, \mathrm{hpT}_{2}, \mathrm{hpT}_{5}\right.$; insets 
Figure 1B-G and Section 3) that were measured in single-molecule burst measurements at a constant temperature $\left(T=21.4 \pm 0.1{ }^{\circ} \mathrm{C}\right)$ and identical buffer conditions. Pulsed-interleaved excitation combined with multiparameter fluorescence detection (MFD) allowed us to separate FRET populations from donor-only, acceptor-only and photobleaching events by the use of several filter algorithms (see Section 3) [62]. Figure 1B-G show the FRET histograms after removal of the above mentioned donor-only, acceptor-only and photobleaching events. As expected, two well-defined peaks representing the open and closed state of the DNA hairpin were measured. FRET events between the folded and unfolded peak do not represent a third static species, but can be identified using methods like a two-channel kernel-based density distribution estimator (FRET-2CDE) for photon distribution analysis, or burst variance analysis (BVA), or a FRET vs. donor lifetime $\left(\tau_{\mathrm{D}(\mathrm{A})}\right)$ plot to be molecules changing their conformation during the diffusion through the confocal volume (see Figure 2 and Section 3).

Figure 2. (A) Scatter plot of FRET-2CDE $v s$. FRET for the DNA hairpin construct $h p B_{5}$. The FRET-2CDE value ( $\tau=45 \mu \mathrm{s}$, kernel time constant) was calculated for every burst. Well-defined closed and opened conformations were selected by the analysis of bursts with a FRET-2CDE value below 15 (threshold indicated by red dashed line); (B) An equivalent method is the Burst variance analysis (BVA). Static, well-defined open or closed species can be found at the shot noise line (black line) within the confidence interval of $99.9 \%$ (grey); (C) FRET vs. $\tau_{\mathrm{D}(\mathrm{A})}$ for the DNA hairpin construct $\mathrm{hpB}_{5}$. The displaced stream between the two FRET states shows that the mid-population in Figure $1 \mathrm{G}$ originates from fast interconverting molecules.
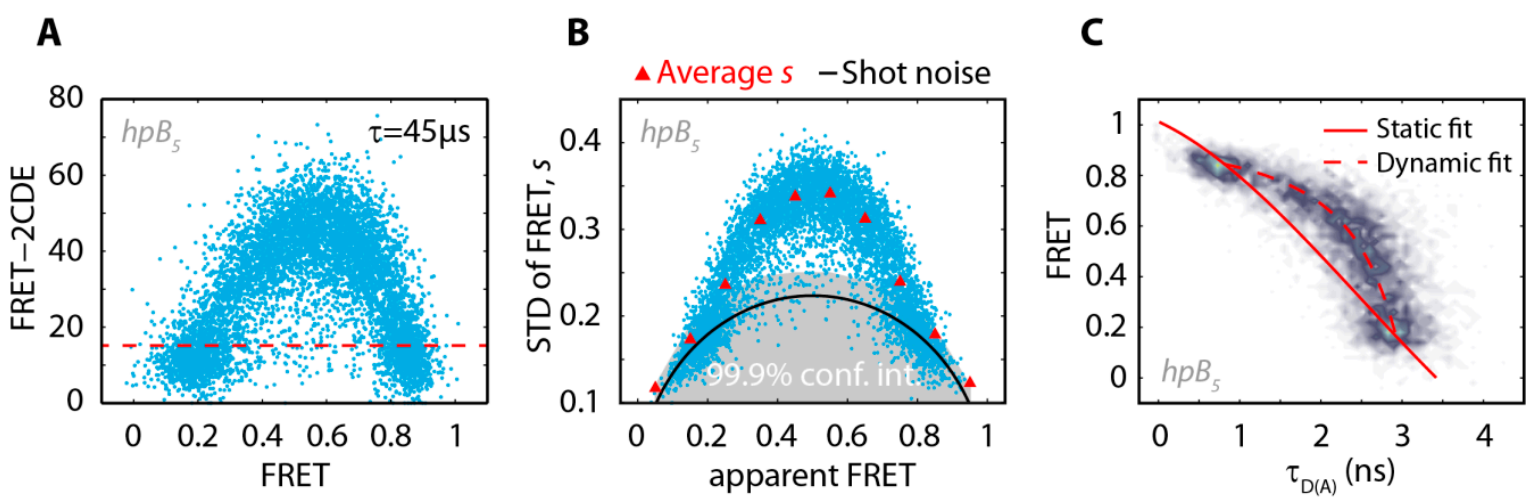

The kinetic bursts were removed using a FRET-2CDE cut-off of 15 (Figure 2A) and the remaining static open and closed populations were fitted with Gaussians to determine the open and closed FRET efficiencies (Table 1).

The FRET efficiency histograms including the kinetic bursts (as shown in Figure 1) were further analyzed with a three-Gaussian (3G) fit [65]. This model assumes two well-defined states (two-state model), which interconvert on a time scale that is comparable to the diffusion time $\left(<t_{\mathrm{D}}>=2 \mathrm{~ms}\right)$ :

$$
E_{\text {open }} \underset{k_{\text {open }}}{\stackrel{k_{\text {close }}}{\rightleftarrows}} E_{\text {close }}
$$


Table 1. The average FRET values with the related standard deviations of the open and closed state for the six different DNA hairpin constructs obtained from $N_{\text {mol }}$ molecules.

\begin{tabular}{cccc}
\hline Substrate & $\boldsymbol{E}_{\text {open }} \pm \boldsymbol{\sigma}_{\text {open }}$ & $\boldsymbol{E}_{\text {close }} \pm \boldsymbol{\sigma}_{\text {close }}$ & $\boldsymbol{N}_{\text {mol }}$ \\
\hline $\mathrm{hpT}_{0}$ & $0.17 \pm 0.05$ & $0.77 \pm 0.05$ & 1622 \\
$\mathrm{hpT}_{2}$ & $0.16 \pm 0.04$ & $0.82 \pm 0.04$ & 1440 \\
$\mathrm{hpT}_{5}$ & $0.19 \pm 0.05$ & $0.85 \pm 0.05$ & 1589 \\
$\mathrm{hpB}_{0}$ & $0.17 \pm 0.04$ & $0.77 \pm 0.04$ & 1058 \\
$\mathrm{hpB}_{2}$ & $0.18 \pm 0.05$ & $0.83 \pm 0.05$ & 1267 \\
$\mathrm{hpB}_{5}$ & $0.19 \pm 0.05$ & $0.86 \pm 0.05$ & 1713 \\
\hline
\end{tabular}

By fitting the FRET histogram with the three-Gaussian distributions that are coupled to each other (see Section 3), opening and closing rates on the millisecond time scale (Table 2) can be extracted. The FRET peak amplitudes reflect the ratio of the opening and closing rate $k_{\text {open }} / k_{\text {close }}$. The FRET efficiency peak between the closed and open state originates from molecules changing their conformation during the diffusion through the confocal volume and, thus, the more area is contained in the center population, the more frequent conformational changes occur.

Table 2. The opening and closing rates of the DNA hairpin constructs, the related changes in Gibbs free energy $\left(\Delta G^{0}\right)$ and the transition state free energy barriers $\left(\Delta G^{\ddagger}\right)$.

\begin{tabular}{cccccc}
\hline Substrate & $\boldsymbol{k}_{\text {close }}\left(\mathbf{m s}^{-\mathbf{1}}\right)$ & $\boldsymbol{k}_{\text {open }}\left(\mathbf{m s}^{-\mathbf{1}}\right)$ & $\left.\Delta \boldsymbol{G}^{\mathbf{0}} \mathbf{( k J} \mathbf{m o l}\right)$ & $\Delta \boldsymbol{G}_{\text {close }}^{\ddagger}(\mathbf{k J} / \mathbf{m o l})$ & $\Delta \boldsymbol{G}_{\text {open }}^{\ddagger}(\mathbf{k J} / \mathbf{m o l})$ \\
\hline $\mathrm{hpT}_{0}$ & $0.86 \pm 0.05$ & $0.20 \pm 0.01$ & $-3.57 \pm 0.26$ & $19.98 \pm 0.13$ & $23.55 \pm 0.11$ \\
$\mathrm{hpT}_{2}$ & $0.92 \pm 0.05$ & $0.29 \pm 0.01$ & $-2.83 \pm 0.22$ & $19.81 \pm 0.13$ & $22.64 \pm 0.12$ \\
$\mathrm{hpT}_{5}$ & $0.91 \pm 0.02$ & $0.35 \pm 0.02$ & $-2.34 \pm 0.19$ & $19.84 \pm 0.06$ & $22.18 \pm 0.12$ \\
$\mathrm{hpB}_{0}$ & $0.66 \pm 0.04$ & $0.41 \pm 0.03$ & $-1.17 \pm 0.33$ & $20.63 \pm 0.15$ & $21.79 \pm 0.18$ \\
$\mathrm{hpB}_{2}$ & $0.87 \pm 0.02$ & $0.55 \pm 0.01$ & $-1.12 \pm 0.10$ & $19.95 \pm 0.06$ & $21.07 \pm 0.05$ \\
$\mathrm{hpB}_{5}$ & $0.90 \pm 0.01$ & $0.84 \pm 0.01$ & $-0.17 \pm 0.06$ & $19.87 \pm 0.03$ & $20.04 \pm 0.04$ \\
\hline
\end{tabular}

Surprisingly, even though the proximal stem of the hairpin was kept identical in the different samples, we observed significant changes in the FRET populations and kinetic rates of the hairpin depending on the labeling strategy and on the gap between the proximal and distal stem (Figure 1, Tables 1 and 2).

\subsection{The Base-Labeled Hairpin is More Dynamic than the Terminal-Labeled Hairpin}

In a first set of analysis, we compared the three terminal-labeled constructs (Figure 1B-D) with the three base-labeled constructs (Figure $1 \mathrm{E}-\mathrm{G}$ ). We found that the FRET efficiencies of the corresponding terminal- and base-labeled hairpins are identical within our resolution for the open and closed populations, respectively (Table 1). This indicates that regardless of the labeling strategy the open and closed states are identical. A striking difference between both labeling strategies can be found in the ratio of the peak amplitudes and the inter-peak FRET efficiency populations. The terminal-labeled constructs (Figure 1B-D) exhibited less dynamics and were predominantly closed while the base-labeled constructs (Figure $1 \mathrm{E}-\mathrm{G}$ ) were more dynamic and resided more often in the open state. 
As expected from the shape of the FRET efficiency distribution, the $3 \mathrm{G}$ analysis revealed that the opening rate of all base-labeled constructs $(\mathrm{hpB})$ are approximately twice as high compared to all terminal-labeled constructs (hpT) (Table 2). This indicates that either hpT is stabilized by the fluorophore attachment at the terminal end or $\mathrm{hpB}$ is destabilized by the C6-linker attached fluorophore to the base.

In contrast, the closing rates are identical among the corresponding hairpins except for $k_{\text {close }}$ of the construct $h \mathrm{hB}_{0}$, which is reduced by $25 \%$ compared to the other closing rates. The closing rate is related to the probability of an end-to-end collision of the DNA hairpin and, thus, is mainly dominated by the loop length and electrostatic repulsion $[43,44]$. In all our constructs the loop length and salt concentration was identical, therefore, we conclude that in the construct $\mathrm{hpB}_{0}$, the end-to-end collision probability is reduced with this labeling strategy.

Overall, the apparent Gibbs free energy differences between the open and closed state are significantly different between the two different labeling strategies with a $\Delta \Delta G^{0}\left(\mathrm{hpT}_{\mathrm{n}}-\mathrm{hpB} \mathrm{B}_{\mathrm{n}}\right) \approx 2 \mathrm{~kJ} / \mathrm{mol}$ (Table 2). Thus, the base-labeled hairpin is generally strongly destabilized compared to the terminal-labeled hairpin.

The closing and opening rates further allowed us to calculate transition state free energies $\left(\Delta G^{*}\right.$ open,

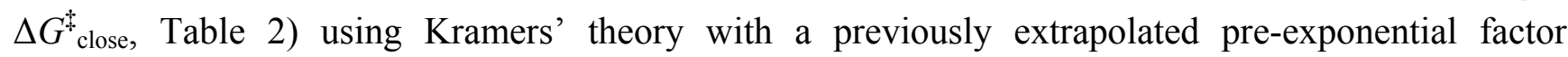
$k_{0}=3 \times 10^{6} \mathrm{~s}^{-1}$ [66] (see Section 3). The free energy barrier heights for closing, $\Delta G^{\ddagger}$ close, are almost identical for the six constructs whereas the barrier heights for opening of the hairpins, $\Delta G^{*}$ open, are varying and decreasing with increasing gap size and are generally lower in the terminal labeled constructs than in the base labeled constructs. Since the thermodynamic stability, $\Delta G^{0}$, is the difference

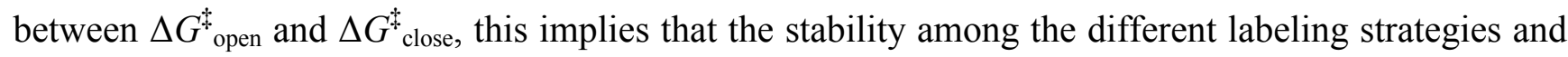
hairpin designs is mainly influenced by the barrier height of the opening process. We compared our experimentally extrapolated barrier heights to a theoretical prediction of the free energy difference for opening of the hairpin $\left(\Delta G^{\ddagger}{ }_{\text {open }}\right)$ as established previously $[16,17]$. To this end, the free energy required for melting a five base pair duplex DNA with our sequence using the nearest-neighbor method [67] (MFOLD, [68,69]) was calculated. The calculation yielded a value of $20 \mathrm{~kJ} / \mathrm{mol}$ for the hybridization free energy matching within error the experimentally determined opening barriers. In a previous study, Nir and coworkers [17] determined barrier heights for opening of a similar hairpin construct that was base labeled and had a gap size of two nucleotides between the proximal stem and the distal stem. They reported experimentally derived barrier heights using Kramers' theory about $0.5-0.7 \mathrm{~kJ} / \mathrm{mol}$ higher than the melting energy of the corresponding duplex using MFOLD. Remarkably, the barrier height of opening of our corresponding construct $\mathrm{hpB}_{2}$ is in good agreement with their offset, however, our construct $\mathrm{hpB}_{5}$ with increased gap size yielded a barrier height that was exactly matching to the calculated MFOLD value of duplex formation. In general, based on the observed differences in the barrier height, the pre-exponential factor can be adjusted to match MFOLD predictions for either of the hairpin constructs as done previously [17], that would yield $k_{0}=2.0 \times 10^{6} \mathrm{~s}^{-1}$ for $\mathrm{hpB}_{2}$ and $k_{0}=3.1 \times 10^{6} \mathrm{~s}^{-1}$ for $\mathrm{hpB}_{5}$. Our data, however, indicates that a small system like this DNA hairpin can be disturbed by the labeling position of the fluorophore leading to potential destabilizations during base labeling or stabilizations due to DNA fluorophore interactions (see Section 2.3). Extrapolations of pre-exponential factors from the experimental data can thus be severely hampered by the sample design and an independent determination of the pre-exponential factor is crucial for further interpretations. 


\subsection{The Acceptor Fluorophore on the Hairpin Interacts with the Double-Stranded Distal Stem}

In a next set of analysis, we determined the influence of the distal stem on the proximal stem by increasing the distance between both stems from zero to two to five nucleotides for both labeling strategies. The FRET efficiencies of the open state did not change significantly (Figure 3A and Table 1). Interestingly, increasing the gap between both stems, led to an increase in the FRET efficiencies for the closed state (Figure 3A and Table 1). The FRET increase is not unexpected due to the reduced persistence length of single-stranded DNA compared to double-stranded DNA and thus a reduced time-averaged end-to-end distance in both constructs.

Figure 3. (A) FRET efficiencies of the low FRET (open) and high FRET (closed) population, with two sigma deviations, for the 5'-modified terminal- and the base-labeled DNA hairpin constructs. The data were extracted from bursts with FRET-2CDE smaller than 15; (B) Related steady state rotational correlation times, obtained from steady state anisotropy fits by the Perrin equation (lines are a guide to the eye).

A

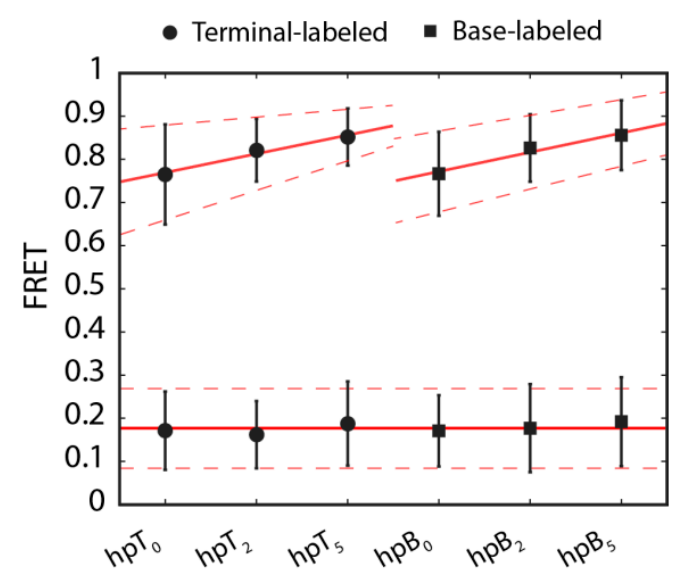

B

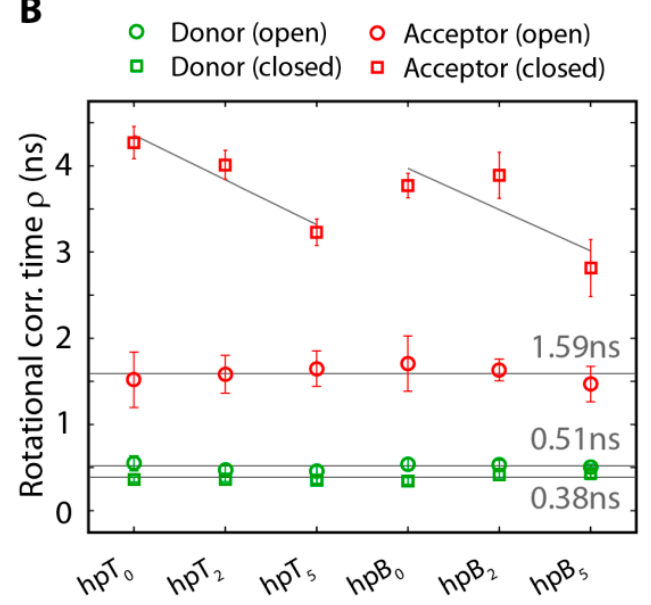

At the same time, for both, terminal-and base labeled hairpins the opening rate $k_{\text {open }}$ increases with the gap size between the proximal and the distal stem (Table 2). The terminal-labeled and the base-labeled constructs with a gap of five nucleotides showed an increase in the opening rate of about $75 \%$ and $100 \%$, respectively, compared to those with a zero nucleotide gap. The increase of the opening rate was in both labeling schemes anti-correlated with the steady state anisotropy rotational correlation time, $\rho_{\mathrm{s}, \mathrm{closed}}$, of the acceptor in the closed state (Table 2 and Figure 3B). Moreover, a detailed global fitting analysis of the steady state anisotropy together with the time-resolved anisotropy exhibited for all constructs a fast and a slow component of the acceptor closed state rotational correlation times, $\rho_{\text {fast }}$ and $\rho_{\text {slow }}$, respectively (exemplarily shown for $\mathrm{hpB}_{5}$ in Figure $4 \mathrm{~A}$, red). The fast decay phase with rotational correlation times of approximately $0.3-0.5 \mathrm{~ns}$ (e.g., $\rho_{\text {fast }}\left(\mathrm{hpB}_{5}\right)=0.33 \pm$ $0.16 \mathrm{~ns}$ ) indicates a freely rotating fluorophore in agreement with the rotational correlation time $\rho_{\mathrm{s}}=0.39 \mathrm{~ns}$ of freely diffusing acceptor molecules (Figure 4A, gray) and values from literature of DNA-bound acceptor molecules [70]. The slow decay time on the other hand indicates a restrained fluorophore with rotational correlation times of $13-19 \mathrm{~ns}$ (e.g., $\left.\rho_{\text {slow }}\left(\mathrm{hpB}_{5}\right)=15 \pm 6 \mathrm{~ns}\right)$. We quantified the relative amplitude, $x_{\text {free }}$, of the fast rotational correlation time of the acceptor in the closed 
conformation for all constructs (Table 3). For both labeling strategies, these fractions were increasing with larger gaps between the proximal and distal stem for both, terminal- and base-labeled hairpin constructs. A larger gap size from zero to five freed the acceptor by approximately $10 \%$. This increase lowers the averaged steady state rotational correlation time significantly by $30 \%$ due to the weight of the slow component of the time-resolved rotational correlation time. The constrained acceptor rotation in the closed hairpin can originate from several factors. A molecular reasoning of this constrain is rather complicated and probably includes several properties of the fluorophore, like hydrophobicity, charge, size, and rigidity of the linker and dye itself. Possible scenarios for the DNA hairpin can be stacking on the terminal basepairs or the interaction with the major groove of the distal stem (limited by the C6 linker length) [56,57,71-73]. This additional interaction can stabilize the closed hairpin structure and thus reduce the opening rate in all constructs.

Figure 4. Global fitting of the steady state (A) and time-resolved anisotropy (B) for $\mathrm{hpB}_{5}$ in the closed conformation (red) reveals two rotational components. The fast decay originates from the freely rotating acceptor dye and the slow decay from the sticking of the acceptor dye to the hairpin. The freely rotating acceptor molecule (gray) shows a steady state rotational correlation time corresponding to the fast component in the hairpin and only a single exponential decay in the time-resolved anisotropy.

A

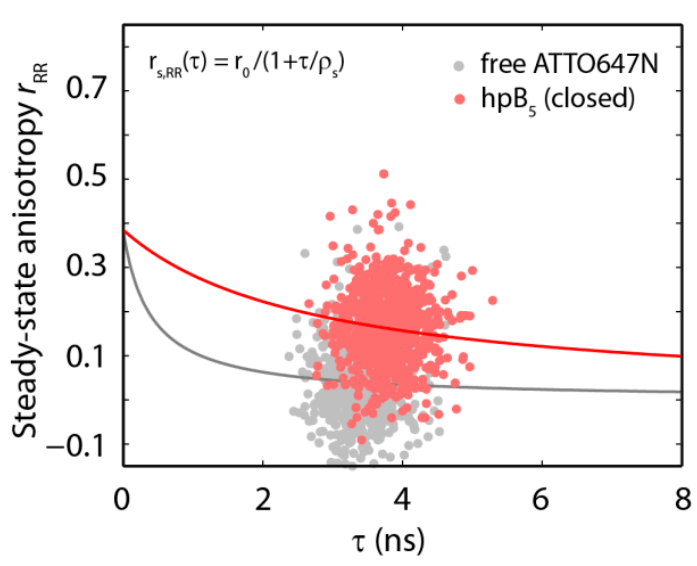

B

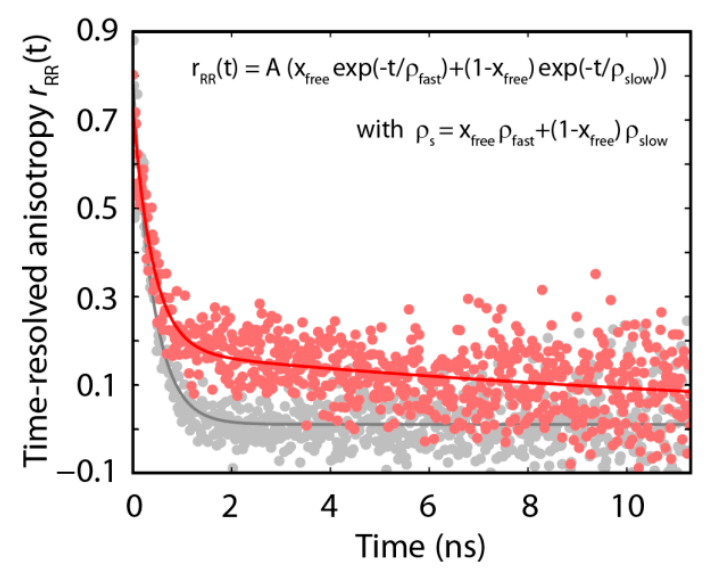

Table 3. Rotational correlation times, relative amplitudes of fast rotational correlation times and the steady state rotational correlation times in the closed hairpin conformation of the acceptor.

\begin{tabular}{ccccc}
\hline Substrate & $\boldsymbol{\rho}_{\text {fast }}(\mathbf{n s})$ & $\boldsymbol{\rho}_{\text {slow }}(\mathbf{n s})$ & $\boldsymbol{x}_{\text {free }}$ & $\boldsymbol{\rho}_{\text {s,closed }}(\mathbf{n s})$ \\
\hline $\mathrm{hpT}_{0}$ & $0.47 \pm 0.11$ & $15 \pm 3$ & $0.74 \pm 0.07$ & $4.27 \pm 0.19$ \\
$\mathrm{hpT}_{2}$ & $0.37 \pm 0.10$ & $14 \pm 3$ & $0.74 \pm 0.06$ & $4.01 \pm 0.17$ \\
$\mathrm{hpT}_{5}$ & $0.51 \pm 0.08$ & $13 \pm 3$ & $0.79 \pm 0.06$ & $3.23 \pm 0.15$ \\
$\mathrm{hpB}_{0}$ & $0.34 \pm 0.09$ & $14 \pm 4$ & $0.76 \pm 0.08$ & $3.77 \pm 0.14$ \\
$\mathrm{hpB}_{2}$ & $0.41 \pm 0.12$ & $19 \pm 8$ & $0.81 \pm 0.08$ & $3.89 \pm 0.27$ \\
$\mathrm{hpB}_{5}$ & $0.33 \pm 0.16$ & $15 \pm 6$ & $0.84 \pm 0.08$ & $2.82 \pm 0.33$ \\
\hline
\end{tabular}

The closing rates of the constructs, on the other hand, were mainly unaffected by the gap size between the proximal and the distal stem, except of the previously mentioned closing rate of $\mathrm{hpB}_{0}$. Here, increasing the gap size from zero nucleotides to two nucleotides $\left(\mathrm{hpB}_{0}->\mathrm{hpB}_{2}\right)$ increased the 
closing rate by $25 \%$ (Table 2$)$. Increasing the gap from two to five nucleotides $\left(\mathrm{hpB}_{2}->\mathrm{hpB}_{5}\right)$ did not further increase the closing rate constant within our experimental resolution. Therefore, a minimal gap of two nucleotides seems to be sufficient to reduce the steric hindrance in the base-labeled construct.

Interestingly, the nearly constant closing rates correlate well with the steady state anisotropy rotational correlation times $\rho_{\mathrm{s}, \text { open }}$ of the acceptor for the open hairpins that were independent of the labeling strategy and constant at a value of around $1.6 \mathrm{~ns}$. Time-resolved anisotropy of the open state revealed again a fast and a slow component with nearly constant ratios and a fast rotational correlation time around $0.4 \mathrm{~ns}\left(\right.$ e.g., $\left.\rho_{\text {fast }}\left(\mathrm{hpB}_{5}\right)=0.37 \pm 0.16 \mathrm{~ns}\right)$ and a slow component of around $4 \mathrm{~ns}$ (e.g., $\left.\rho_{\text {slow }}\left(\mathrm{hpB}_{5}\right)=4 \pm 1 \mathrm{~ns}\right)$.

The microenvironment of the donor fluorophore was unaffected by hairpin opening and closing. All constructs yielded a donor lifetime of $\tau_{\mathrm{D}(0)}=(3.43 \pm 0.03) \mathrm{ns}$, and the steady state anisotropy rotational correlation time for the open and closed conformation $\rho_{\mathrm{s}, \mathrm{open}}=0.51 \mathrm{~ns}$ and $\rho_{\mathrm{s}, \text { closed }}=0.38 \mathrm{~ns}$, respectively, was constant.

\section{Experimental Section}

\subsection{DNA Hairpin Design and Labeling Strategies}

Fluorescently labeled and HPLC-purified DNA single strands were obtained from IBA (Göttingen, Germany) modified with the corresponding $N$-hydroxysuccinimidyl ester (NHS) donor and acceptor fluorophore derivatives of ATTO532 and ATTO647N (ATTO-TEC, Siegen, Germany). The acceptor was conjugated with NHS-chemistry to the thymidine on the 5'-end of the top strands either via terminal or base labeling (see Figure 5).

Figure 5. Chemical drawings of the acceptor labeling strategies. (A) In terminal labeling the acceptor is coupled to the amino-C6-modified 5'-phosphate-thymidine; (B) In base labeling the acceptor is attached to an amino-C6-modified thymine.

A

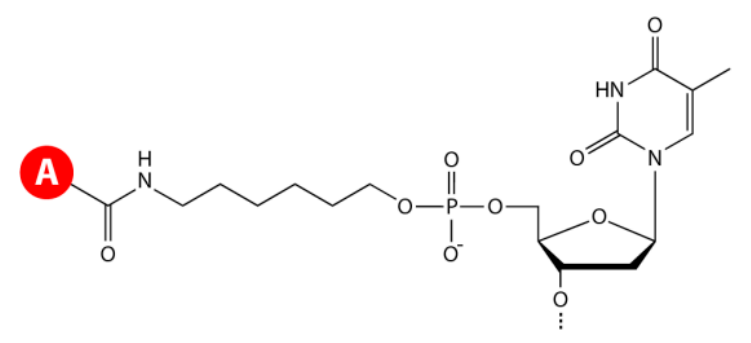

A ATT0647N

B

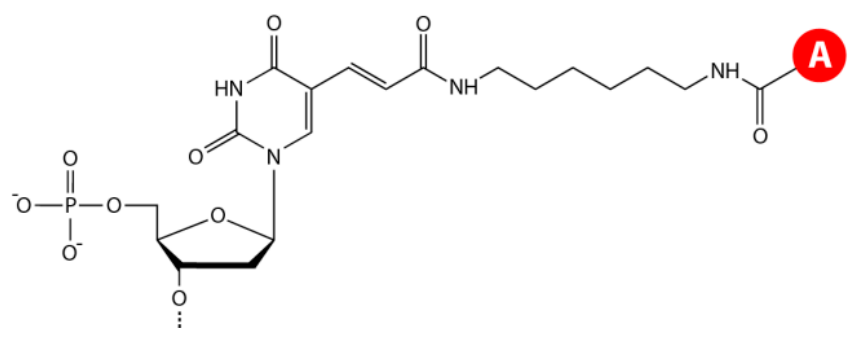

In terminal labeling, the acceptor was covalently linked to the amino-C6-modified 5'-phosphate whereas in base labeling the acceptor was attached to an amino-C6-modified thymidine (dTC6). The donor was labeled at position 26 of the bottom strands via NHS-chemistry to the dTC6 base. Top 
strand $\mathrm{hpT}_{0,2,5}:$ 5'-ATTO647N-TGGTT-(T) 21 $_{1}$-AACCATTCTTCACAAACCAGTCCAAACTATCAA AACTTA-3'; Top strand hpB ${ }_{0,2,5}:$ 5'-dTC6-NH-(ATTO647N)-GGTT-(T) ${ }_{21}$ AACCATTCTTCACAAA CCAGTCCAAACTATCACAAACTTA-3'; Bottom strand hpt $\mathrm{h}_{0} / \mathrm{hpB}_{0}$ : 5'-TAAGTTTGTGATAGTTT GGACTGGTdTC6-NH-(ATTO532)-TGTGAAGAA-3'; Bottom strand hpT 2 / hpB 2 : 5'-TAAGTTTGT GATAGTTTGGACTGGTdTC6-NH-(ATTO532)-TGTGAAG-3'; Bottom strand hpt hpB $_{5}$ : 5'-TA AGTTTGTGATAGTTTGGACTGGT dTC6-NH-(ATTO532)-TGTG-3'.

The DNA hairpin constructs were annealed at a concentration of $5 \mu \mathrm{M}$ in $10 \mathrm{mM}$ Tris- $\mathrm{HCl} \mathrm{pH} 8.0$ (Roth, Karlsruhe, Germany), $50 \mathrm{mM} \mathrm{NaCl}$ (Roth) and $1 \mathrm{mM}$ EDTA (AMRESCO, Solon, OH, USA) by heating to $90^{\circ} \mathrm{C}$ and cooling to room temperature in $1 \mathrm{~K} / \mathrm{min}$ steps.

\section{2. smFRET Measurements and Analysis Procedures}

Freely diffusing single molecule measurements were performed in a $20 \mathrm{mM}$ Tris-HCl (Roth) $\mathrm{pH} 8$ buffer, containing $250 \mu \mathrm{M}$ EDTA (AMRESCO), $175 \mathrm{mM} \mathrm{NaCl}$ (Roth). To minimize blinking of the fluorophores an aged saturated Trolox-solution was added to the buffer. The Trolox-solution (6-hydroxy-2,5,7,8-teramethylchroman-2-carboxylic acid, Sigma-Aldrich, St. Louis, MI, USA) was prepared freshly and aged over night at $4{ }^{\circ} \mathrm{C}$ [74]. Multiple molecule detection was avoided by a very diluted (5 pM) hairpin solution and samples were measured in a Methoxy-PEG (5 kDa, Rapp Polymere, Tübingen, Germany) passivated home-built flow-chamber [75]. During the measurements the temperature of the sample was controlled by objective cooling to avoid environmental effects on the melting behaviour of the DNA. The temperature of the slide was monitored with a $230 \mu \mathrm{m}$-sized temperature probe (Physitemp Instruments, Clifton, NJ, USA) attached to the top of the chamber. The temperature remained stable with $\Delta T<0.2 \mathrm{~K}$ for all measurements. The individual values of the mean temperatures, for each measurement, are shown in Table 4.

Table 4. Mean temperature of the single molecule measurements.

\begin{tabular}{cc}
\hline Substrate & $\boldsymbol{T}\left({ }^{\circ} \mathbf{C}\right)$ \\
\hline $\mathrm{hpT}_{0}$ & $21.34 \pm 0.09$ \\
$\mathrm{hpT}_{2}$ & $21.34 \pm 0.07$ \\
$\mathrm{hpT}_{5}$ & $21.41 \pm 0.09$ \\
$\mathrm{hpB}_{0}$ & $21.36 \pm 0.15$ \\
$\mathrm{hpB}_{2}$ & $21.34 \pm 0.07$ \\
$\mathrm{hpB}_{5}$ & $21.38 \pm 0.14$ \\
\hline
\end{tabular}

FRET experiments were performed in a home-built confocal microscope combined with Time-Correlated Single Photon Counting (TCSPC) (Figure 6). For the donor and acceptor excitation we used two pulsed laser sources with wavelengths of $530 \mathrm{~nm}$ and $640 \mathrm{~nm}$ (LDH-P-FA-530L and LDH-D-C-640, Picoquant, Berlin, Germany). The two lasers were driven in the pulsed interleaved excitation (PIE) mode (PDL828 "Sepia II", Picoquant) with a total repetition rate of $50 \mathrm{MHz}$ and a delay time of $20 \mathrm{~ns}$ and a laser power of $178 \mu \mathrm{W}$ for the green laser and $156 \mu \mathrm{W}$ for the red laser measured before the objective. The dye emission was collected $70 \mu \mathrm{m}$ in solution by a $60 \mathrm{x}$ water objective (CFI Plan Apo 60x Water, Nikon, Tokyo, Japan) with a numerical aperture of 1.2. For the separation of illumination and detection pathway, we used a dual-edge dichroic mirror (zt532/640rpc, 
Chroma, Bellows Falls, VT, USA) and for the separation of the donor and acceptor emission, after a polarizing beam splitter (PBS), a single-edge dichroic mirror (FF650-Di01, Semrock, New York, NY, USA). Emitted photons were bandpass filtered (F1: FF01-582/75, Semrock; F2: ET700/75M, Chroma), detected by four single-photon avalanche diodes ( $\tau$-SPADs, Picoquant) and registered by four independent channels of a TCSPC module (HydraHarp 400, Picoquant).

Figure 6. Schematic illustration of the home-built confocal TCSPC microscope. Donor and acceptor fluorophores were excited at $530 \mathrm{~nm}$ and $640 \mathrm{~nm}$ with pulsed-interleaved excitation (PIE) with a total repetition rate of $50 \mathrm{MHz}$ to remove donor only and acceptor only molecules from the analysis. Donor and acceptor anisotropy and fluorescence lifetime are measured simultaneously to obtain information about the microenvironment and rotational freedom of the fluorophores. A stoichiometry vs. FRET plot ( $S$ - $E$-plot) allows the identification of FRET molecules (orange), donor-only molecules (green) and acceptor-only molecules (red). PBS: polarizing beam splitter; APD: avalanche photo diode; F1/F2: bandpass filter.

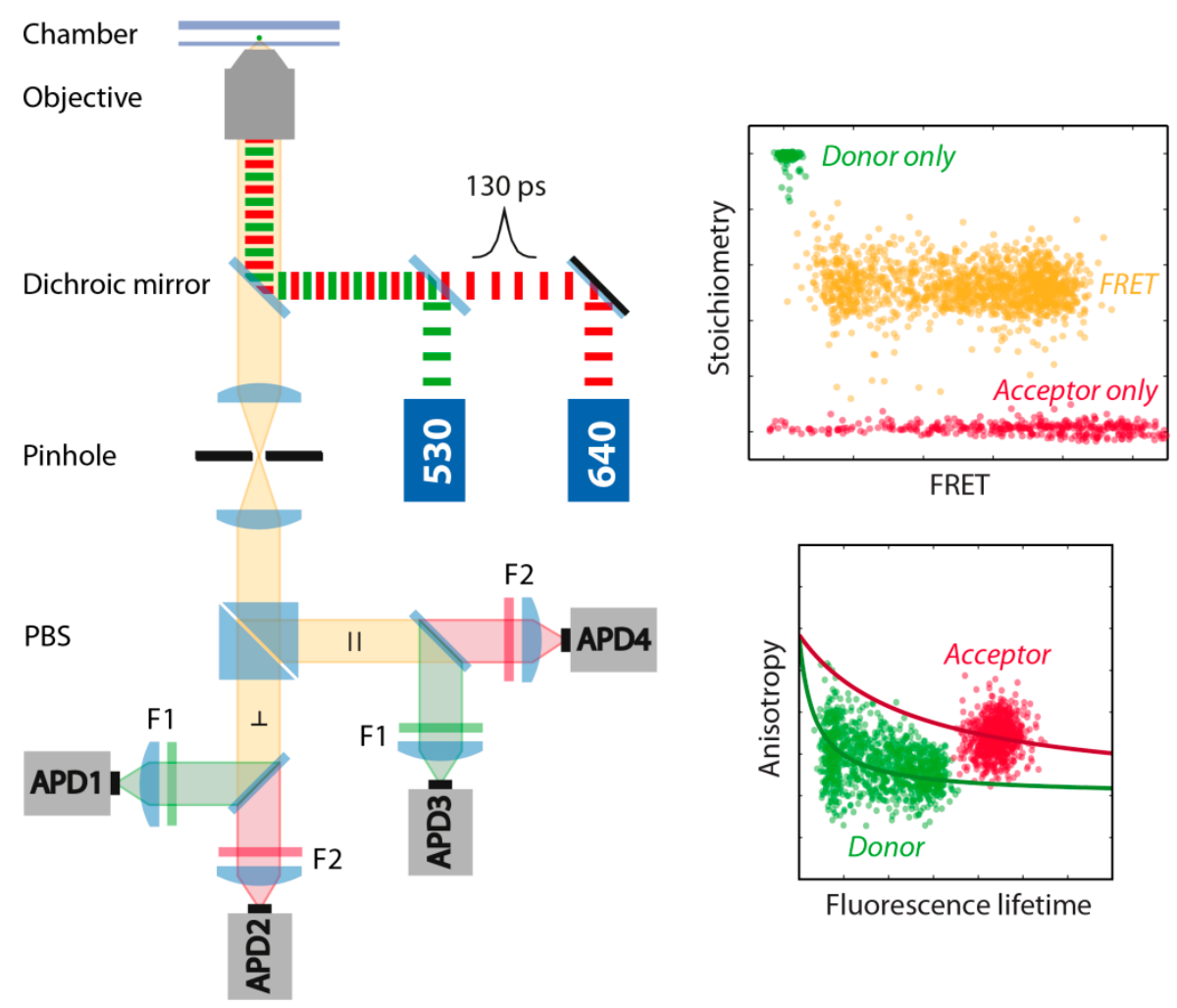

From the acquired photon stream only bursts with an inter-photon time smaller than $25 \mu \mathrm{s}$ and a minimum total number of 200 photons were selected. To remove molecules affected by photobleaching, an asymmetric burst filter was applied, which discards all bursts with an absolute difference of the burst-averaged macroscopic photon arrival time measured in the acceptor channel after acceptor excitation and the burst-averaged macroscopic photon arrival time measured in the donor and acceptor channel after donor excitation $\left|T_{R R}-T_{G, G+R}\right|$ bigger than $50 \mu \mathrm{s}$ [62]. For the remaining molecules the corrected stoichiometry and FRET values, $S$ and $E$, respectively, were derived from burst intensities as follows: 


$$
\begin{gathered}
S=\frac{F_{G R}-\alpha F_{R R}-\beta F_{G G}+\gamma F_{G G}}{F_{G R}-\alpha F_{R R}-\beta F_{G G}+\gamma F_{G G}+F_{R R}} \\
E=\frac{F_{G R}-\alpha F_{R R}-\beta F_{G G}}{F_{G R}-\alpha F_{R R}-\beta F_{G G}+\gamma F_{G G}}
\end{gathered}
$$

Here, $F_{\mathrm{GG}}$ and $F_{\mathrm{GR}}$ are the background subtracted fluorescence intensities in the donor and the acceptor channel, respectively, after donor excitation and $F_{R R}$ in the acceptor channel after acceptor excitation. The detection correction factor $\gamma$ and the correction factors for direct excitation, $\alpha$, and crosstalk, $\beta$, were extracted from the gained $S-E$ plot $(\alpha=0.038, \beta=0.014$ and $\gamma=0.7)$ [62]. Only bursts with a stoichiometry between 0.3 and 0.7 were used for further analysis.

Population averaged steady state rotational correlation times, time-resolved rotational correlation times and fluorescence lifetimes were determined as described [62,63]. Both, the steady state anisotropy and the time-resolved anisotropy, were fitted globally using the Perrin equation and a double-exponential decay, respectively (see insets Figure 4).

We used a two-channel kernel-based density distribution estimator (2CDE) for photon distribution analysis, to separate static molecules from those that undergo multiple transitions during diffusion through the detection spot [15]. After calculating the FRET-2CDE value ( $\tau=45 \mu \mathrm{s}$, kernel time constant) for every molecule, static FRET bursts could be easily separated from dynamic bursts by applying a threshold of 15 (Figure 2A). Additionally, we used burst variance analysis (BVA) [13] as an alternative method to resolve dynamic bursts from static bursts (Figure 2B). To identify FRET fluctuations within a burst the standard deviation of the mean FRET efficiency is calculated for individual molecules with a five photon binning of the burst trace. Molecules with an underlying FRET distribution in their burst trace show an increased standard deviation $s$, (see Figure 2B) whereas static FRET species can be found at $\sigma_{E}=\sqrt{E(1-E) / n}$ with $n=5$, the number of photons.

Kinetic rates were extracted from FRET histograms using the three-Gaussian approximation for a two-state system described by Gopich and Szabo [65]. The three-Gaussian function is defined by the FRET efficiencies of the open and closed state $E_{\text {open }}$ and $E_{\text {close, }}$, the opening and the closing rate $k_{\text {open }}$ and $k_{\text {close }}$, the bin time $T$ and the average of the inverse of the total number of photons $\left\langle N^{-1}\right\rangle$. The average of the inverse of the total photons and the bin time were derived from the measurement, whereas $E_{\text {open }}, E_{\text {close }}, k_{\text {open }}$ and $k_{\text {close }}$ were freely varied. The FRET states and the conversion rates were obtained from a global fit to a set of three FRET efficiency histograms generated from the same measurement but using different bin times within a burst: $0.5 \mathrm{~ms}, 1 \mathrm{~ms}$ and $1.5 \mathrm{~ms}$ (see Figure 7). Bin size artefacts and errors of least-squares fits were minimized by a FRET bin size of 0.001 .

The Gibbs free energy difference was derived from:

$$
\Delta G^{0}=-R T \ln \left(\frac{k_{\text {close }}}{k_{\text {open }}}\right)
$$

with $R$ being the universal gas constant, $T$ the absolute temperature and $k_{\text {open }}$ and $k_{\text {close }}$ are the opening and closing rates, respectively (see Table 2 ). 
Figure 7. Global fit of the DNA hairpin construct $h p T_{0}$ using the three-Gaussian approximation [65]. The three FRET efficiency histograms were obtained from different bin times: $0.5 \mathrm{~ms}(\mathbf{A}), 1 \mathrm{~ms}(\mathbf{B})$ and $1.5 \mathrm{~ms}(\mathbf{C})$. A small FRET bin size of 0.001 was chosen to minimize bin size artefacts.
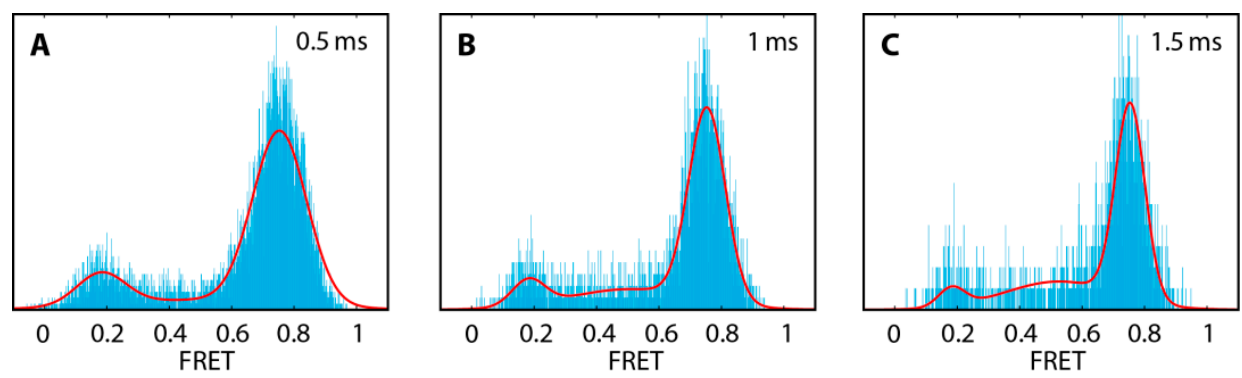

The height of the Gibbs free energy barriers for opening and closing of the hairpins in a one-dimensional free energy profile was calculated using Kramers' transition-state theory (see Table 2):

$$
\Delta G_{\text {open } / \text { close }}^{\ddagger}=-R T \ln \left(\frac{k_{\text {open } / \text { close }}}{k_{0}}\right)
$$

where $k_{\text {open }}$ and $k_{\text {close }}$ are the observed opening and closing rates, respectively, $\Delta G_{\text {open }}^{\ddagger}$ and $\Delta G^{\ddagger}$ close are the free energy barrier heights with respect to the free energies of the closed and the open state, respectively, and the pre-exponential factor $k_{0}$, reflecting the transition rate in the absence of a free energy barrier. In our calculations, we used the previously extrapolated pre-exponential factor $k_{0}=3 \times 10^{6} \mathrm{~s}^{-1}[66]$.

\section{Conclusions}

In this study, we showed that the opening and closing rates of a millisecond folding hairpin, monitored by smFRET, are strongly influenced by the labeling scheme of the proximal stem and the distance to the adjacent distal stem. While the microenvironment of the donor, attached to the distal stem, is identical within our experimental error, the acceptor fluorophore was a major determinant in affecting the folding kinetics of the hairpin. The kinetic analysis revealed that base-labeled hairpins are destabilized compared to the terminal-labeled hairpins. This weakening could originate from a disturbed adenine-thymine bond by the fluorophore attached to the thymine base. On the other hand, we observed a stabilizing effect of the closed conformation, which decreased with increasing gap size between the proximal and distal stem. Analysis of the time-resolved anisotropy of the acceptor dye suggests that this originates from a dye-DNA interaction. Increasing the gap distance between both stems led to an increase in the fraction of freely rotating acceptor. Furthermore, we found that the base-labeling in the $\mathrm{hpB}_{0}$ construct reduced the closing kinetics of the hairpin significantly, most likely due to steric hindrance. It is important to note, that in all our studies the inherent hairpin structure of the loop length and the five annealing bases was unchanged, however, the deduced apparent free energy between the closed and open state changed significantly from $\Delta G^{0}\left(\mathrm{hpT}_{0}\right)=-3.6 \mathrm{~kJ} / \mathrm{mol}$ to $\Delta G^{0}\left(\mathrm{hpB}_{5}\right)=-0.2 \mathrm{~kJ} / \mathrm{mol}$. Our data using multi-parameter fluorescence detection of fluorophore anisotropy indicate that the constructs $\mathrm{hpT}_{5}$ and $\mathrm{hpB}_{5}$ reflect the least disturbed systems. With the assumption that an undisturbed system is the most stable system, the $\mathrm{hpT}_{5}$ construct would be the 
experimental candidate of choice. On the other hand, in light of the match of the theoretical barrier height for opening and the experimentally extrapolated barrier height the $\mathrm{hpB}_{5}$ construct would be the preferred hairpin.

Previous studies showed that the choice of the FRET pair severely affects the information deduced from single-molecule FRET experiments [14]. We found that an additional important factor for the design of single-molecule FRET experiments is also the labeling scheme. Especially studies that analyze biomolecular kinetics on the millisecond timescale, e.g., DNA or RNA hairpin formation, protein folding, conformational changes in enzymes, are prone to be significantly affected by destabilizing and stabilizing effects of fluorophores attached to the biomolecule. Thus, it is crucial to vary FRET pairs and the attachment chemistry to minimize the effect of the labeling chemistry. Taking these precautions into account for the design of experiments, single-molecule FRET remains one of the most powerful techniques to study structure-function relationships and underlying energy landscapes of biomolecules.

\section{Acknowledgments}

The authors highly appreciate discussions and experimental insights from Don Lamb (LudwigMaximilians-Universität München, Germany) and group members. We further thank all group members of the Schlierf group, in particular Marko Swoboda for helpful comments on the manuscript. This work was supported by the German Federal Ministry of Education and Research BMBF 03Z2EN11 and 03Z2ES1 (to M.S.) and a scholarship by the Stipendienstiftung Rheinland-Pfalz (to. G.K.).

\section{Author Contributions}

A.H., G.K. and M.S. designed research. A.H. performed and A.H. and G.K. analyzed experiments. A.H. provided analytical tools. A.H., G.K. and M.S. discussed results and wrote the manuscript.

\section{Conflicts of Interest}

The authors declare no conflict of interest.

\section{References}

1. Kapanidis, A.N.; Strick, T. Biology one molecule at a time. Trends Biochem. Sci. 2009, 34, 234-243.

2. Joo, C.; Balci, H.; Ishitsuka, Y.; Buranachai, C.; Ha, T. Advances in single-molecule fluorescence methods for molecular biology. Annu. Rev. Biochem. 2008, 77, 51-76.

3. Tan, E.; Wilson, T.J.; Nahas, M.K.; Clegg, R.M.; Lilley, D.M.J.; Ha, T. A four-way junction accelerates hairpin ribozyme folding via a discrete intermediate. Proc. Natl. Acad. Sci. USA 2003, 100, 9308-9313.

4. Yeoman, J.A.; Orte, A.; Ashbridge, B.; Klenerman, D.; Balasubramanian, S. RNA conformation in catalytically active human telomerase. J. Am. Chem. Soc. 2010, 132, 2852-2853.

5. Dupuis, N.F.; Holmstrom, E.D.; Nesbitt, D.J. Molecular-crowding effects on single-molecule RNA folding/unfolding thermodynamics and kinetics. Proc. Natl. Acad. Sci. USA 2014, 111, 8464-8469. 
6. Zhuang, X.; Bartley, L.E.; Babcock, H.P.; Russell, R.; Ha, T.; Herschlag, D.; Chu, S. A single-molecule study of RNA catalysis and folding. Science 2000, 288, 2048-2051.

7. McKinney, S.A.; Freeman, A.D.J.; Lilley, D.M.J.; Ha, T. Observing spontaneous branch migration of Holliday junctions one step at a time. Proc. Natl. Acad. Sci. USA 2005, 102, 5715-5720.

8. Zhuang, X.; Kim, H.; Pereira, M.J.B.; Babcock, H.P.; Walter, N.G.; Chu, S. Correlating structural dynamics and function in single ribozyme molecules. Science 2002, 296, 1473-1476.

9. Wallace, M.I.; Ying, L.; Balasubramanian, S.; Klenerman, D. FRET Fluctuation spectroscopy: Exploring the conformational dynamics of a DNA hairpin loop. J. Phys. Chem. B 2000, 104, 11551-11555.

10. Grunwell, J.R.; Glass, J.L.; Lacoste, T.D.; Deniz, A.A.; Chemla, D.S.; Schultz, P.G. Monitoring the conformational fluctuations of DNA hairpins using single-pair fluorescence resonance energy transfer. J. Am. Chem. Soc. 2001, 123, 4295-4303.

11. Nir, E.; Michalet, X.; Hamadani, K.M.; Laurence, T.A.; Neuhauser, D.; Kovchegov, Y.; Weiss, S. Shot-noise limited single-molecule FRET histograms: Comparison between theory and experiments. J. Phys. Chem. B 2006, 110, 22103-22124.

12. Santoso, Y.; Torella, J.P.; Kapanidis, A.N. Characterizing single-molecule FRET dynamics with probability distribution analysis. ChemPhysChem 2010, 11, 2209-2219.

13. Torella, J.P.; Holden, S.J.; Santoso, Y.; Hohlbein, J.; Kapanidis, A.N. Identifying molecular dynamics in single-molecule FRET experiments with burst variance analysis. Biophys. J. 2011, $100,1568-1577$.

14. Kügel, W.; Muschielok, A.; Michaelis, J. Bayesian-inference-based fluorescence correlation spectroscopy and single-molecule burst analysis reveal the influence of dye selection on DNA hairpin dynamics. ChemPhysChem 2012, 13, 1013-1022.

15. Tomov, T.E.; Tsukanov, R.; Masoud, R.; Liber, M.; Plavner, N.; Nir, E. Disentangling subpopulations in single-molecule FRET and ALEX experiments with photon distribution analysis. Biophys. J. 2012, 102, 1163-1173.

16. Tsukanov, R.; Tomov, T.E.; Berger, Y.; Liber, M.; Nir, E. Conformational dynamics of DNA hairpins at millisecond resolution obtained from analysis of single-molecule FRET histograms. J. Phys. Chem. B 2013, 117, 16105-16109.

17. Tsukanov, R.; Tomov, T.E.; Masoud, R.; Drory, H.; Plavner, N.; Liber, M.; Nir, E. Detailed study of DNA hairpin dynamics using single-molecule fluorescence assisted by DNA origami. J. Phys. Chem. B 2013, 117, 11932-11942.

18. Müller, B.K.; Reuter, A.; Simmel, F.C.; Lamb, D.C. Single-pair FRET characterization of DNA tweezers. Nano Lett. 2006, 6, 2814-2820.

19. Cisse, I.I.; Kim, H.; Ha, T. A rule of seven in Watson-Crick base-pairing of mismatched sequences. Nat. Struct. Mol. Biol. 2012, 19, 623-627.

20. Bokinsky, G.; Rueda, D.; Misra, V.K.; Rhodes, M.M.; Gordus, A.; Babcock, H.P.; Walter, N.G.; Zhuang, X. Single-molecule transition-state analysis of RNA folding. Proc. Natl. Acad. Sci. USA 2003, 100, 9302-9307.

21. Tomov, T.E.; Tsukanov, R.; Liber, M.; Masoud, R.; Plavner, N.; Nir, E. Rational design of DNA motors: Fuel optimization through single-molecule fluorescence. J. Am. Chem. Soc. 2013, 135, 11935-11941. 
22. Nahas, M.K.; Wilson, T.J.; Hohng, S.; Jarvie, K.; Lilley, D.M.J.; Ha, T. Observation of internal cleavage and ligation reactions of a ribozyme. Nat. Struct. Mol. Biol. 2004, 11, 1107-1113.

23. Schuler, B.; Lipman, E.A.; Eaton, W.A. Probing the free-energy surface for protein folding with single-molecule fluorescence spectroscopy. Nature 2002, 419, 743-747.

24. Margittai, M.; Widengren, J.; Schweinberger, E.; Schröder, G.F.; Felekyan, S.; Haustein, E.; König, M.; Fasshauer, D.; Grubmüller, H.; Jahn, R.; et al. Single-molecule fluorescence resonance energy transfer reveals a dynamic equilibrium between closed and open conformations of syntaxin 1. Proc. Natl. Acad. Sci. USA 2003, 100, 15516-15521.

25. Diez, M.; Zimmermann, B.; Börsch, M.; König, M.; Schweinberger, E.; Steigmiller, S.; Reuter, R.; Felekyan, S.; Kudryavtsev, V.; Seidel, C.A.M.; et al. Proton-powered subunit rotation in single membrane-bound F0F1-ATP synthase. Nat. Struct. Mol. Biol. 2004, 11, 135-141.

26. Majumdar, D.S.; Smirnova, I.; Kasho, V.; Nir, E.; Kong, X.; Weiss, S.; Kaback, H.R. Single-molecule FRET reveals sugar-induced conformational dynamics in LacY. Proc. Natl. Acad. Sci. USA 2007, 104, 12640-12645.

27. Santoso, Y.; Joyce, C.M.; Potapova, O.; le Reste, L.; Hohlbein, J.; Torella, J.P.; Grindley, N.D.F.; Kapanidis, A.N. Conformational transitions in DNA polymerase I revealed by single-molecule FRET. Proc. Natl. Acad. Sci. USA 2010, 107, 715-720.

28. Marcinowski, M.; Höller, M.; Feige, M.J.; Baerend, D.; Lamb, D.C.; Buchner, J. Substrate discrimination of the chaperone $\mathrm{BiP}$ by autonomous and cochaperone-regulated conformational transitions. Nat. Struct. Mol. Biol. 2011, 18, 150-158.

29. Borgia, A.; Wensley, B.G.; Soranno, A.; Nettels, D.; Borgia, M.B.; Hoffmann, A.; Pfeil, S.H.; Lipman, E.A.; Clarke, J.; Schuler, B. Localizing internal friction along the reaction coordinate of protein folding by combining ensemble and single-molecule fluorescence spectroscopy. Nat. Commun. 2012, 3, doi:10.1038/ncomms2204.

30. Chung, H.S.; McHale, K.; Louis, J.M.; Eaton, W.A. Single-molecule fluorescence experiments determine protein folding transition path times. Science 2012, 335, 981-984.

31. Soranno, A.; Buchli, B.; Nettels, D.; Cheng, R.R.; Müller-Späth, S.; Pfeil, S.H.; Hoffmann, A.; Lipman, E.A.; Makarov, D.E.; Schuler, B. Quantifying internal friction in unfolded and intrinsically disordered proteins with single-molecule spectroscopy. Proc. Natl. Acad. Sci. USA 2012, 109, 17800-17806.

32. Sikor, M.; Mapa, K.; von Voithenberg, L.V.; Mokranjac, D.; Lamb, D.C. Real-time observation of the conformational dynamics of mitochondrial Hsp70 by spFRET. EMBO J. 2013, 32, 1639-1649.

33. Hohlbein, J.; Aigrain, L.; Craggs, T.D.; Bermek, O.; Potapova, O.; Shoolizadeh, P.; Grindley, N.D.F.; Joyce, C.M.; Kapanidis, A.N. Conformational landscapes of DNA polymerase I and mutator derivatives establish fidelity checkpoints for nucleotide insertion. Nat. Commun. 2013, 4, doi:10.1038/ncomms3131.

34. Chung, H.S.; Eaton, W.A. Single-molecule fluorescence probes dynamics of barrier crossing. Nature 2013, 502, 685-688.

35. Ratzke, C.; Hellenkamp, B.; Hugel, T. Four-colour FRET reveals directionality in the Hsp90 multicomponent machinery. Nat. Commun. 2014, 5, doi:10.1038/ncomms5192.

36. Roy, R.; Hohng, S.; Ha, T. A practical guide to single-molecule FRET. Nat. Methods 2008, 5, $507-516$. 
37. Schuler, B.; Hofmann, H. Single-molecule spectroscopy of protein folding dynamics_-Expanding scope and timescales. Curr. Opin. Struct. Biol. 2013, 23, 36-47.

38. Bikard, D.; Loot, C.; Baharoglu, Z.; Mazel, D. Folded DNA in action: Hairpin formation and biological functions in prokaryotes. Microbiol. Mol. Biol. Rev. 2010, 74, 570-588.

39. Bevilacqua, P.C.; Blose, J.M. Structures, kinetics, thermodynamics, and biological functions of RNA hairpins. Annu. Rev. Phys. Chem. 2008, 59, 79-103.

40. Kortmann, J.; Narberhaus, F. Bacterial RNA thermometers: Molecular zippers and switches. Nat. Rev. Microbiol. 2012, 10, 255-265.

41. Tan, W.; Wang, K.; Drake, T.J. Molecular beacons. Curr. Opin. Chem. Biol. 2004, 8, 547-553.

42. Tyagi, S.; Kramer, F.R. Molecular beacons: Probes that fluoresce upon hybridization. Nat. Biotechnol. 1996, 14, 303-308.

43. Bonnet, G.; Krichevsky, O.; Libchaber, A. Kinetics of conformational fluctuations in DNA hairpin-loops. Proc. Natl. Acad. Sci. USA 1998, 95, 8602-8606.

44. Bonnet, G.; Tyagi, S.; Libchaber, A.; Kramer, F.R. Thermodynamic basis of the enhanced specificity of structured DNA probes. Proc. Natl. Acad. Sci. USA 1999, 96, 6171-6176.

45. Wallace, M.I.; Ying, L.; Balasubramanian, S.; Klenerman, D. Non-Arrhenius kinetics for the loop closure of a DNA hairpin. Proc. Natl. Acad. Sci. USA 2001, 98, 5584-5589.

46. Jung, J.; van Orden, A. Folding and unfolding kinetics of DNA hairpins in flowing solution by multiparameter fluorescence correlation spectroscopy. J. Phys. Chem. B 2005, 109, 3648-3657.

47. Kim, J.; Doose, S.; Neuweiler, H.; Sauer, M. The initial step of DNA hairpin folding: A kinetic analysis using fluorescence correlation spectroscopy. Nucleic Acids Res. 2006, 34, 2516-2527.

48. Torres, T.; Levitus, M. Measuring conformational dynamics: A new FCS-FRET approach. J. Phys. Chem. B 2007, 111, 7392-7400.

49. Jung, J.; Ihly, R.; Scott, E.; Yu, M.; van Orden, A. Probing the complete folding trajectory of a DNA hairpin using dual beam fluorescence fluctuation spectroscopy. J. Phys. Chem. B 2008, 112, 127-133.

50. Li, H.; Ren, X.; Ying, L.; Balasubramanian, S.; Klenerman, D. Measuring single-molecule nucleic acid dynamics in solution by two-color filtered ratiometric fluorescence correlation spectroscopy. Proc. Natl. Acad. Sci. USA 2004, 101, 14425-14430.

51. Jung, J.; van Orden, A. A three-state mechanism for DNA hairpin folding characterized by multiparameter fluorescence fluctuation spectroscopy. J. Am. Chem. Soc. 2006, 128, 1240-1249.

52. Di Fiori, N.; Meller, A. The Effect of dye-dye interactions on the spatial resolution of single-molecule FRET measurements in nucleic acids. Biophys. J. 2010, 98, 2265-2272.

53. Zanetti-Domingues, L.C.; Tynan, C.J.; Rolfe, D.J.; Clarke, D.T.; Martin-Fernandez, M. Hydrophobic fluorescent probes introduce artifacts into single molecule tracking experiments due to non-specific binding. PLoS One 2013, 8, e74200.

54. Hwang, H.; Kim, H.; Myong, S. Protein induced fluorescence enhancement as a single molecule assay with short distance sensitivity. Proc. Natl. Acad. Sci. USA 2011, 108, 7414-7418.

55. Lee, W.; von Hippel, P.H.; Marcus, A.H. Internally labeled Cy3/Cy5 DNA constructs show greatly enhanced photo-stability in single-molecule FRET experiments. Nucleic Acids Res. 2014, $42,5967-5977$. 
56. Iqbal, A.; Arslan, S.; Okumus, B.; Wilson, T.J.; Giraud, G.; Norman, D.G.; Ha, T.; Lilley, D.M.J. Orientation dependence in fluorescent energy transfer between Cy3 and Cy5 terminally attached to double-stranded nucleic acids. Proc. Natl. Acad. Sci. USA 2008, 105, 11176-11181.

57. Sanborn, M.E.; Connolly, B.K.; Gurunathan, K.; Levitus, M. Fluorescence properties and photophysics of the sulfoindocyanine Cy3 linked covalently to DNA. J. Phys. Chem. B 2007, 111, 11064-11074.

58. Ha, T.; Tinnefeld, P. Photophysics of fluorescent probes for single-molecule biophysics and super-resolution imaging. Annu. Rev. Phys. Chem. 2012, 63, 595-617.

59. Luo, G.; Wang, M.; Konigsberg, W.H.; Xie, X.S. Single-molecule and ensemble fluorescence assays for a functionally important conformational change in T7 DNA polymerase. Proc. Natl. Acad. Sci. USA 2007, 104, 12610-12615.

60. Müller, B.K.; Zaychikov, E.; Bräuchle, C.; Lamb, D.C. Pulsed interleaved excitation. Biophys. J. 2005, 89, 3508-3522.

61. Kapanidis, A.N.; Lee, N.K.; Laurence, T.A.; Doose, S.; Margeat, E.; Weiss, S. Fluorescence-aided molecule sorting: Analysis of structure and interactions by alternating-laser excitation of single molecules. Proc. Natl. Acad. Sci. USA 2004, 101, 8936-8941.

62. Kudryavtsev, V.; Sikor, M.; Kalinin, S.; Mokranjac, D.; Seidel, C.A.M.; Lamb, D.C. Combining MFD and PIE for accurate single-pair Förster resonance energy transfer measurements. ChemPhysChem 2012, 13, 1060-1078.

63. Sisamakis, E.; Valeri, A.; Kalinin, S.; Rothwell, P.J.; Seidel, C.A.M. Accurate single-molecule FRET studies using multiparameter fluorescence detection. Methods Enzymol. 2010, 475, 455-514.

64. Chung, H.S.; Gopich, I.V.; McHale, K.; Cellmer, T.; Louis, J.M.; Eaton, W.A. Extracting rate coefficients from single-molecule photon trajectories and FRET efficiency histograms for a fastfolding protein. J. Phys. Chem. A 2011, 115, 3642-3656.

65. Gopich, I.V; Szabo, A. FRET efficiency distributions of multistate single molecules. J. Phys. Chem. B 2010, 114, 15221-15226.

66. Woodside, M.T.; Behnke-Parks, W.M.; Larizadeh, K.; Travers, K.; Herschlag, D.; Block, S.M. Nanomechanical measurements of the sequence-dependent folding landscapes of single nucleic acid hairpins. Proc. Natl. Acad. Sci. USA 2006, 103, 6190-6195.

67. SantaLucia, J. A unified view of polymer, dumbbell, and oligonucleotide DNA nearest-neighbor thermodynamics. Proc. Natl. Acad. Sci. USA 1998, 95, 1460-1465.

68. Zuker, M. Mfold web server for nucleic acid folding and hybridization prediction. Nucleic Acids Res. 2003, 31, 3406-3415.

69. Markham, N.R.; Zuker, M. DINAMelt web server for nucleic acid melting prediction. Nucleic Acids Res. 2005, 33 (Suppl. 2), W577-W581.

70. Sindbert, S.; Kalinin, S.; Nguyen, H.; Kienzler, A.; Clima, L.; Bannwarth, W.; Appel, B.; Müller, S.; Seidel, C.A.M. Accurate distance determination of nucleic acids via Förster resonance energy transfer: Implications of dye linker length and rigidity. J. Am. Chem. Soc. 2011, 133, 2463-2480.

71. Spiriti, J.; Binder, J.K.; Levitus, M.; van der Vaart, A. Cy3-DNA stacking interactions strongly depend on the identity of the terminal basepair. Biophys. J. 2011, 100, 1049-1057. 
72. Unruh, J.R.; Gokulrangan, G.; Lushington, G.H.; Johnson, C.K.; Wilson, G.S. Orientational dynamics and dye-DNA interactions in a dye-labeled DNA aptamer. Biophys. J. 2005, 88, 3455-3465.

73. Gokulrangan, G.; Unruh, J.R.; Holub, D.F.; Ingram, B.; Johnson, C.K.; Wilson, G.S. DNA aptamer-based bioanalysis of IgE by fluorescence anisotropy. Anal. Chem. 2005, 77, 1963-1970.

74. Cordes, T.; Vogelsang, J.; Tinnefeld, P. On the mechanism of Trolox as antiblinking and antibleaching reagent. J. Am. Chem. Soc. 2009, 131, 5018-5019.

75. Swoboda, M.; Henig, J.; Cheng, H.-M.; Brugger, D.; Haltrich, D.; Plumeré, N.; Schlierf, M. Enzymatic oxygen scavenging for photostability without $\mathrm{pH}$ drop in single-molecule experiments. ACS Nano 2012, 6, 6364-6369.

Sample Availability: Samples of the compounds are available from the authors.

(C) 2014 by the authors; licensee MDPI, Basel, Switzerland. This article is an open access article distributed under the terms and conditions of the Creative Commons Attribution license (http://creativecommons.org/licenses/by/3.0/). 